\title{
CRY1 Regulates Chemoresistance in Association With NANOG by Inhibiting Apoptosis via STAT3 Pathway in Patients With Cervical Cancer
}

\author{
GWAN HEE HAN ${ }^{1,2}$, JULIE KIM ${ }^{3}$, HEE YUN ${ }^{4}$, HANBYOUL CHO ${ }^{5,6,7}$, \\ JOON-YONG CHUNG ${ }^{7}$, JAE-HOON KIM ${ }^{5,6}$ and STEPHEN M. HEWITT ${ }^{7}$ \\ ${ }^{1}$ Department of Obstetrics and Gynecology, Kyung Hee University Hospital at Gangdong, Seoul, Republic of Korea; \\ ${ }^{2}$ Yonsei University Graduate School, Seoul, Republic of Korea; \\ ${ }^{3}$ Weill Cornell Medical College, New York, NY, U.S.A.; \\ ${ }^{4}$ Department of Obstetrics and Gynecology, Gangnam Severance Hospital, \\ Yonsei University College of Medicine, Seoul, Republic of Korea; \\ ${ }^{5}$ Department of Obstetrics and Gynecology, Yonsei University College of Medicine, Seoul, Republic of Korea; \\ ${ }^{6}$ Institute of Women's Life Medical Science, Yonsei University College of Medicine, Seoul, Republic of Korea; \\ ${ }^{7}$ Laboratory of Pathology, Center for Cancer Research, National Cancer Institute, \\ National Institutes of Health, Bethesda, MD, U.S.A.
}

\begin{abstract}
Background/Aim: Cryptochrome 1 (CRY1), a core circadian gene, modulates circadian rhythm and carcinogenesis. Here, we investigated the role of CRY1 and its correlation with NANOG, a stem cell transcription factor, in cervical cancer. Materials and Methods: Immunohistochemistry with tissue microarray was performed to evaluate CRYI and NANOG expression in cervical cancer tissues, and their functional roles were assessed in cervical cancer cell lines. Results: CRYI or NANOG was significantly over-expressed in cervical cancer tissues. Notably, combined over-expression of CRY1 and NANOG was correlated with a significantly poor OS and DFS and showed a stronger predictive value for chemoradiation response than each single protein. Furthermore, siCRYI induced apoptosis, decreased NANOG expression, suppressed STAT3 signalling, and activated p53 signalling in cervical cancer cell lines. Conclusion: CRYI and NANOG overexpression serves as a strong predictive biomarker for prognosis and chemoradiation response, and may be a new therapeutic target in patients with cervical cancer.
\end{abstract}

This article is freely accessible online.

Correspondence to: Hanbyoul Cho, MD, Ph.D., Professor, Department of Obstetrics and Gynecology, Gangnam Severance Hospital, Yonsei University College of Medicine, 211 Eonju-Ro, Gangnam-Gu, Seoul 06273, Republic of Korea. Tel: +82 220193430, Fax: +82 234628209, e-mail: hanbyoul@yuhs.ac

Key Words: CRY1, NANOG, apoptosis, cervical cancer, biomarker, chemoradiation response.
Cervical cancer is the second most common gynecological cancer with a prevalence of $6.6 \%$. It accounts for the second highest rate of cancer-related deaths among gynecological cancers, and had a 7.5\% mortality worldwide in 2018 (1). Although vaccination against the human papillomavirus and cytological screening systems have been investigated, its incidence remains high, and nearly $50 \%$ of patients with cervical cancer are diagnosed at the advanced stage. Concurrent chemoradiation therapy (CCRT) followed by radical hysterectomy in early-stage cervical cancer often results in a satisfactory survival rate. However, patients with advanced stage disease often experience relapse, and the survival rate in the first 5 years remains poor. Therefore, there is an urgent need to determine biomarkers to predict the prognosis and treatment efficacy, which would subsequently improve patient outcomes for locally-advanced cervical cancer cases and aid in candidate selection for treatment strategies, especially CCRT.

The circadian rhythm is an endogenous time-keeping mechanism comprising autonomous peripheral oscillators that are coordinated by a master oscillator located in the suprachiasmatic nucleus of the hypothalamus, and various peripheral clocks that are in the organs to coordinate sleep, metabolism, and immunity at appropriate times during the day (6-8). Accumulating evidence indicate that the disruption of circadian rhythms because of an irregular lifestyle or genetic alterations is associated with pathologic consequences (9). For example, epidemiological studies have suggested that the disruption of the circadian clock due to chronic sleep deprivation, jet lag, or shift work increases the incidence of cancer risk (10). Alterations in circadian-related 
genes fundamentally disrupt basic cellular physiology, which in turn, increases susceptibility to diseases such as cancer by controlling cell metabolism, proliferation, and apoptosis (1113). To date, 14 circadian clock genes, which are present in most human body cells, have been identified; these genes interact to create a network with multiple feedback loops at the transcriptional or translational level (14). Among them, cryptochrome $1(C R Y 1)$ is a key circadian clock gene that plays an important role in tumorigenesis. A previous study by Habashy et al. (15) has reported the association between epigenetic silencing of $C R Y 1$ and an indolent clinical course in chronic lymphocytic leukaemia (CLL) patients. Besides CLL, an association between CRY1 over-expression and tumour progression and poor prognosis has been observed in patients with colorectal cancer, renal cancer, and osteosarcoma (16-18).

Tumorigenesis involves complex and diversified processes, which include inhibiting apoptosis as a signal generated via cell contact, developing new blood vessels to supply oxygen and nutrition to support tumour growth, and inducing the capacity to migrate, invade, and cause chemotherapeutic resistance $(19,20)$. To date, many studies have indicated crucial genes involved in apoptosis, which show periodic patterns of expression. Mutation in $C R Y$ has also been shown to sensitize a $p 53$ mutant and enhance apoptosis by interfacing with many signalling pathways involved in cancer development.

However, the association of $C R Y 1$ with the response to chemotherapy and carcinogenesis in cervical cancer remains unclear. Therefore, in the present study, we investigated the association of $C R Y 1$ with clinicopathological features, its prognostic significance in cervical cancer, and its functional roles in association with response to chemotherapy. Moreover, as cancer stem cell functions are regulated by circadian oscillation, we investigated the correlation between $N A N O G$, a stem cell transcription factor, and $C R Y 1$ in cervical cancer.

\section{Materials and Methods}

Patients and tumour samples. A total of 188 cervical cancer specimens, 318 high-grade cervical intraepithelial neoplasia (CIN) specimens, 102 low-grade CIN, and 270 normal non-adjacent cervical epithelial tissues were collected from patients who underwent either radical hysterectomy or conisation at the Department of Obstetrics and Gynecology, Gangnam Severance Hospital, Yonsei University College of Medicine between March 1996 and March 2010. Additional formalin-fixed, paraffinembedded (FFPE) blocks were provided by the Korea Gynecologic Cancer Bank under the Bio \& Medical Technology Development Program of the Ministry of the National Research Foundation (NRF) funded by the Korean government (MIST) (NRF2017M3A9B8069610). After performing a pathology review of all the tumour specimens, adequate specimens were included in the present study. The clinicopathological characteristics, including age, survival time, survival status, tumour size, grade, cell types, presence of lymph node (LN) metastasis or lymphovascular space invasion, and response to CCRT were obtained from the pathology and medical reports. Cervical cancer staging was performed according to the International Federation of Gynaecology and Obstetrics (FIGO) staging system, and tumour grade was determined as per the World Health Organisation grading. Patients who were eligible for surgical resection underwent type 3 radical hysterectomy with pelvic LN dissection; CCRT was performed after the surgery in patients with a risk of relapse, positive LNs, parametrial invasion, or positive resection margins. Response to therapy was evaluated according to the response evaluation criteria in solid tumours (RECIST; version 1.1) using either computed tomography or magnetic resonance imaging. The present study was approved by the Institutional Review Board (IRB) of Gangnam Severance Hospital (IRB no. 3-2020-0377).

Tissue microarray construction and immunohistochemistry analysis. After constructing tissue microarrays (TMAs) with $1 \mathrm{~mm}$ cores from each archival FFPE block, the TMAs were serially cut into 5 $\mu \mathrm{m}$ sections, and then sections were deparaffinized and rehydrated with xylene, followed by washes with ethanol and distilled water, and analysed by immunohistochemical (IHC) staining. Then, the sections were treated with $3 \%$ hydrogen peroxide for $10 \mathrm{~min}$ to quench the endogenous peroxidase activity, and heat-activated antigen retrieval was performed using an antigen retrieval buffer of pH 6 (Dako, Carpinteria, CA, USA) in a steam pressure cooker (Pascal; Dako) for $20 \mathrm{~min}$. The sections were then treated with a protein block (Dako) for 10 min to block nonspecific staining. Next, the sections were incubated with the primary antibodies anti-CRY1 (rabbit polyclonal antibody, Cat. \# PAB4717, 1:500; Abnova, Taipei, Taiwan) and anti-NANOG (rabbit antibody, clone \# D7364, 1:200; Cell Signalling Technology, Danvers, MA, USA), for $1 \mathrm{~h}$ in Dako Autostainer Plus (Dako). For the antigen-antibody reaction, the sections were incubated with Dako EnVision+ Dual Link SystemHRP (Dako) and 3,3'-diaminobenzidine (DAB; Dako). Finally, haematoxylin was used to lightly counterstain the TMA slide, after which, the slides were examined by a light microscope.

Evaluation of IHC staining. The high-resolution optical scanner Nanozoomer 2.0 HT (Hamamatsu Photonics K.K., Hamamatsu, Japan) was used to scan the slide after IHC staining. Then, Visiopharm Integrator System v6.5.0.2303 (VIS; Visiopharm, Hørsholm, Denmark), a digital image analysis software, was used to quantify the staining intensity after training the system with digitally painted image examples. The brown-coloured (DAB) intensity of each defined image was quantified and categorized as follows: 3 , strong; 2 , moderate; 1 , weak; and 0 , negative. The immunostaining score was determined by multiplying the staining intensity (0-3) and percentage (0-100) of a positive cell.

Cell culture. Human cervical cancer cell lines, Caski and SNU-17, were purchased from Korea Cell Line Bank (Seoul, Republic of Korea). The cells were cultured in RPMI 1640 supplemented with $10 \%$ fetal bovine serum (FBS) and $1 \%$ penicillin/streptomycin in a humidified atmosphere with $5 \% \mathrm{CO}_{2}$ at $37^{\circ} \mathrm{C}$.

siRNA transfection. Specific small interfering RNAs (siRNAs) for CRY1 and NANOG, and control siRNA (siControl) were purchased from Bioneer (Daejeon, Republic of Korea). The siRNA sequences were as follows: CRY1 \#1 5'-CUCUGUCUGAUGACCAUGA-3' 
(sense); 5'-UCAUGGUCAUCAGACAGAG-3' (antisense); CRY1 \#2 5'-CUCUGUUAACACAGACAGU-3' (sense); 5'-ACUGUCUGU GUUAACAGAG-3' (antisense); NANOG \#1 5'-AGUGUUUCAAU GAGU-3' (sense); 5'-ACUCAUUGAAACACU-3' (antisense); NANOG \#2 5'-UCUCGUAUUUGCUGC-3' (sense); and 5'GCAGCAAAUACGAGA-3' (antisense). siRNA was transfected into cells grown in a 6-well plate at a dose of 100 pmol per well using Lipofectamine ${ }^{\circledR}$ RNAiMAX Reagent (Invitrogen, Gaithersburg, MD, USA) as per the manufacturer's instructions. After $72 \mathrm{~h}$ of transfection, the siRNA-treated cells were collected for western blotting analysis.

Western blotting analysis. After harvesting the cells, cells were lysed with cold cell lysis buffer (Cell Signalling Technology) containing phenylmethylsulphonyl fluoride. Cellular proteins were resolved by sodium dodecyl sulphate-polyacrylamide gel electrophoresis and transferred onto a nitrocellulose membrane. Antibodies against CRY1, c-Myc, p53, and $\alpha$-actinin were purchased from Santa Cruz Biotechnology (Santa Cruz, CA, USA). Antibodies against NANOG, PARP, caspase-3, phosphoSTAT3Tyr705, and STAT3 were purchased from the Cell Signalling Technology. The visualization of immunoreactive bands was performed by using chemiluminescence reagents (Thermo Fisher Scientific, Waltham, MA, USA). Each experiment was repeated three times.

Cell proliferation assay. Cell proliferation was measured using an EZ-Cytox assay kit (Daeil Lab Service, Seoul, Republic of Korea) as per the manufacturer's instructions. The absorbance in each well was measured at $450 \mathrm{~nm}$ using a microplate reader (Bio-Rad Laboratories, Inc., Hercules, CA, USA). Each experiment was repeated three times.

Colony formation assay. Caski and SNU-17 cells were transfected with siRNAs targeting CRYI or NANOG. After $24 \mathrm{~h}$ of transfection, the cells $(500$ cells/well) were seeded in a 6 -well plate and maintained in an incubator at $37^{\circ} \mathrm{C}$ with $5 \% \mathrm{CO}_{2}$ for 2 weeks. The cells were then fixed with methanol for $10 \mathrm{~min}$ and stained with $0.5 \%$ crystal violet for $30 \mathrm{~min}$. After rinsing with distilled water, the colonies formed in each well were counted using a microscope. Each experiment was repeated three times.

Boyden chamber assay. The 48-wells microchemotaxis chambers (Neuro Probe, Gaithersburg, MD, USA) were used to examine cell invasion. Culture medium with $10 \%$ FBS was added to each well of the bottom chamber, and the Matrigel (BD Biosciences, San Jose, CA, USA)-coated membranes (\#PFB8; Neuro Probe) was gently placed over the walls of the bottom chamber. siRNA-transfected cells $\left(1 \times 10^{5}\right.$ cells $/ 50 \mu \mathrm{l}$ of medium containing $0.05 \%$ FBS) were seeded in the upper chambers. After $48 \mathrm{~h}$, the membranes were fixed and stained with a Diff-quik solution (Sysmex, Kobe, Japan), and the uninvaded cells were removed from the upper surface of the membrane. The invading cells were counted with using Axio Imager M2 microscope (Carl Zeiss, Thornwood, NY, USA) in six random high-power fields per filter. Each experiment was repeated three times.

Flow cytometric analysis. Caski and SNU-17 cells were treated for $48 \mathrm{~h}$ with cisplatin, then collected for apoptosis analyses. The cells were incubated with Annexin V-FITC (BD Biosciences, Waltham, MA, USA) and propidium iodide (PI) for 15 min at room temperature in the dark. After staining the samples with Annexin V-FITC and PI, stained cells were diluted using the binding buffer and immediately analysed by a flow cytometer. Flow cytometric analyses were performed on a FACS-Canto II (BD Biosciences) analyser, followed by analysis using FACSDiva software (BD Biosciences).

Statistical analysis. Mann-Whitney or Kruskal-Wallis tests were performed for statistical analysis of CRY1 and NANOG expression levels, as appropriate. Pearson's chi-square test was used when necessary. Kaplan-Meier method was used for plotting the overall survival (OS) and disease-free survival (DFS) curves. Cox proportional hazard model for the univariate and multivariate models was used for survival analysis. SPSS version 25.0 (SPSS Inc., Chicago, IL, USA) was used for statistical analysis, and $p<0.05$ was set as the criterion for evaluating significance.

\section{Results}

CRY1 and NANOG protein expression in cervical cancer. To elucidate the clinical role of CRY1 and NANOG in cervical cancer, we assessed CRY1 and NANOG expression by IHC using TMAs of 188 cervical cancer tissues, 318 high-grade CIN samples, 102 low-grade CIN specimens, and 270 nonadjacent normal epithelial tissues. From these, 144 cervical cancer tissues, 193 high-grade CIN samples, 73 low-grade CIN samples, and 226 non-adjacent normal epithelial tissues could be interpreted for CRY1 and 170 cervical cancer tissues, 265 high-grade CIN samples, 87 low-grade CIN samples, and 270 non-adjacent normal epithelial tissues could be interpreted for NANOG; the rest were excluded from IHC analysis because of the complexity and staining status of the samples. Representative IHC figures of CRY1 and NANOG expression are shown in Figure 1A. As shown in Figure 1B and Table I, the expression of CRY1 was higher in the cervical cancer tissues than in the high-grade CIN, low-grade CIN, and non-adjacent normal epithelial tissues (all $p<0.001$; Table I and Figure 1B). Over-expression of CRY1 (CRY1+) was significantly related with advanced FIGO stage $(p=0.009$; Table I and Figure 1B) and poor chemoradiation response ( $p=0.003$; Table I). The expression of NANOG was significantly higher in the cervical cancer tissues than in the high-grade CIN, low-grade CIN, and non-adjacent normal epithelial tissues (all $p<0.001$; Table I and Figure 1B), and was associated with poor chemoradiation response $(p=0.035$; Table I and Figure 1B). These results indicate that CRY1 and NANOG expression may be valuable biomarkers for therapeutic response in patients with cervical cancer.

As accumulating evidence suggests that disruption of the circadian rhythm in cancer stem cells might contribute to disease progression and chemoradiation resistance, we further analysed the chemoradiation response when both CRY1 and NANOG were over-expressed. Combined CRY1+/NANOG+ expression showed a higher predictive value for chemoradiation response than did expression of each protein alone, that is, either CRY1+ or NANOG+ expression $(p<0.001$; Figure 1C). 


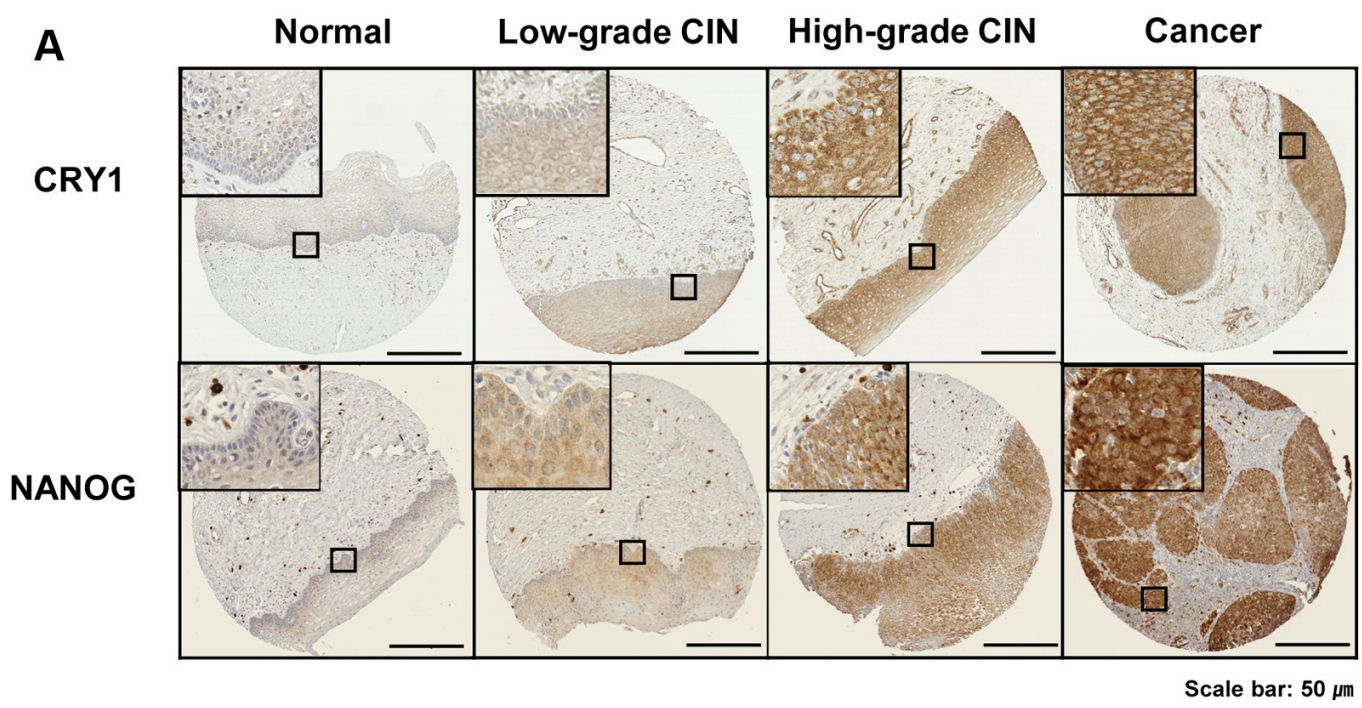

B
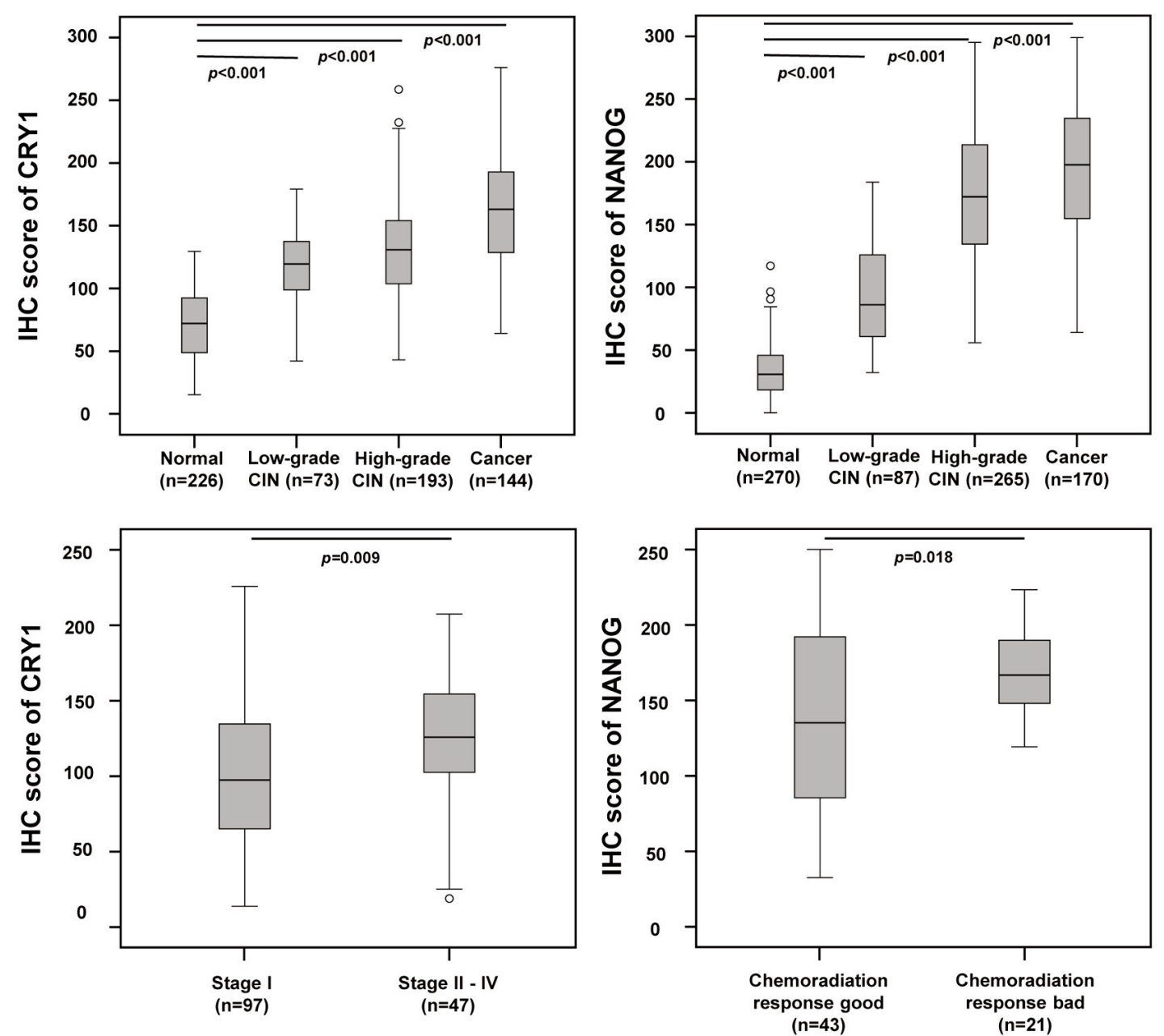

Figure 1. Continued 

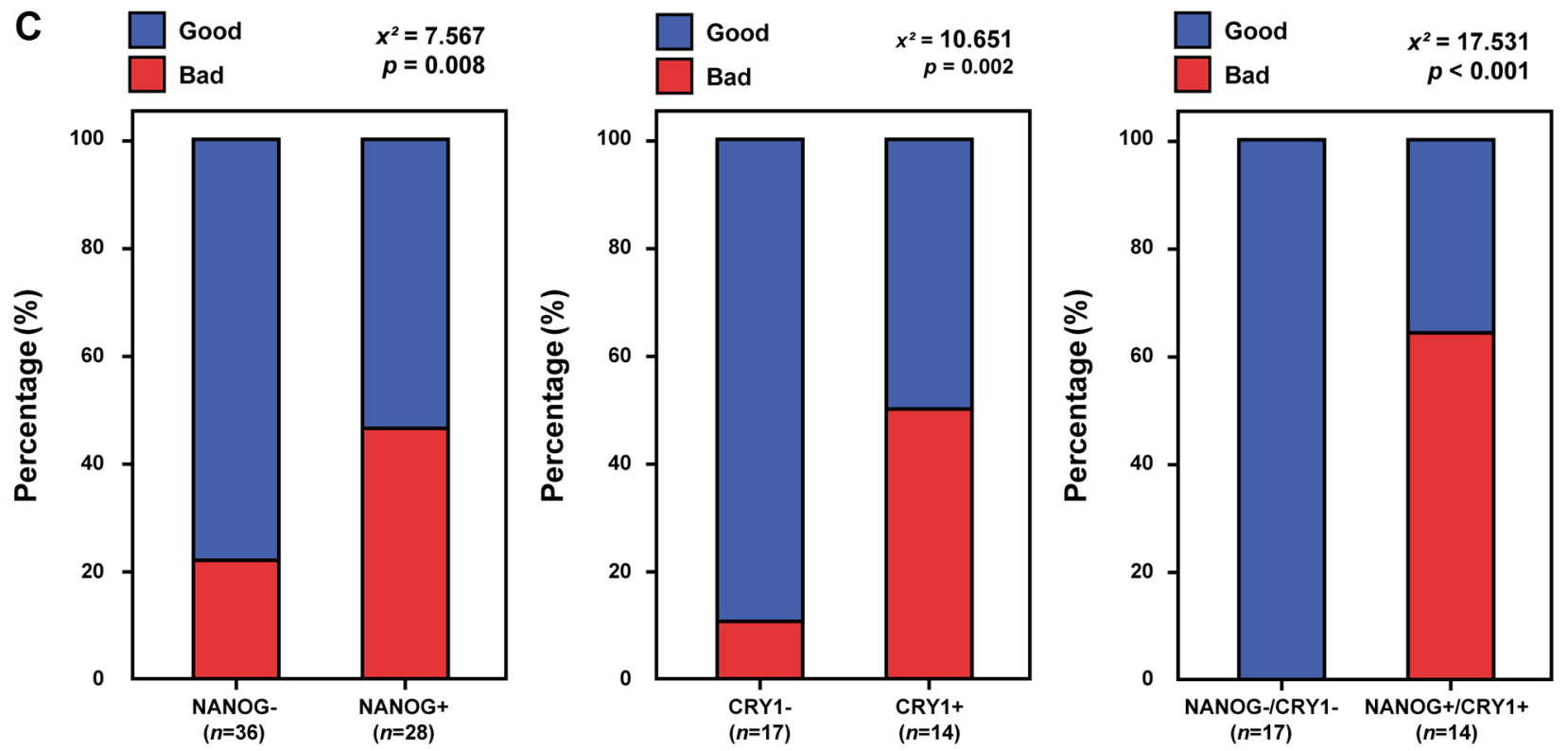

Figure 1. CRY1 and NANOG expression in normal cervical epithelial, low-grade cervical intraepithelial neoplasia (CIN), high-grade CIN, and cervical cancer tissues. (A) Representative immunohistochemical images of CRY1 and NANOG in normal cervical epithelial, low-grade CIN, highgrade CIN, and cervical cancer tissues. Scale bar: $50 \mu \mathrm{m}(B)$ Comparison of IHC staining score for CRY1 in normal cervical epithelial, low-grade CIN, high-grade CIN, and cervical cancer tissues and between stage I and stages II-IV in cervical cancer tissues. Comparison of IHC scoring for NANOG in normal cervical epithelial, low-grade CIN, high-grade CIN, and cervical cancer tissues and between good and poor chemoradiation response in cervical cancer patients. (C) Combined over-expression of CRY1 and NANOG showed more predictive value for the response to chemoradiation treatment over single gene expression of CRY1 or NANOG.

Prognostic significance of CRY1 and NANOG expression. We next evaluated the prognostic value of CRY1 or NANOG expression in association with survival outcomes of patients with cervical cancer. Patients with CRY1+ expression were significantly associated with poor OS and DFS compared to those with low expression of CRY1 (CRY1-; both $p=0.001$; Figure $2 \mathrm{~A}$ and D). NANOG+ expression was also significantly associated with poor OS and DFS compared to low expression of NANOG (NANOG-; both $p=0.001$; Figure 2B and E).

In addition to evaluating the expression of a single protein for prognosis, we compared the OS and DFS of patients with CRY1+/NANOG+ and CRY1-/NANOG- expression. Significantly poorer OS and DFS were observed in patients with CRY1+/NANOG+ expression than in patients with CRY1-/NANOG- expression ( $p<0.001$; Figure $2 \mathrm{C}$ and F). Cox proportional univariate and multivariate analyses were used for further analysis of OS and DFS, and though the expression of each CRY1 and NANOG separately served as independent prognostic factors for OS and DFS, the combined marker, CRY1+/NANOG+ expression, was most strongly related to poor OS and DFS in cervical cancer [hazard ratio $(\mathrm{HR})=11.17,95 \%$ confidence interval $(\mathrm{CI})=1.36-91.16, p=0.024$; and $\mathrm{HR}=18.68$, 95\%CI $=2.34$ 149.00, $p=0.006$; Table II].
CRY1 and NANOG knock-down in cervical cancer cells. To characterize its functional role in cervical cancer, $C R Y 1$ was silenced using siRNA that targeted CRYI (siCRYl) in Caski and SNU-17 cells. CRY1 knock-down was verified using western blotting analysis (Figure 3A). Subsequently, we examined the effect of $C R Y 1$ knock-down on cervical cancer cell growth in vitro. The cell proliferation curve showed that $C R Y 1$ knock-down significantly inhibited proliferation of both Caski and SNU-17 cells (Figure 3B). The effect of CRYI on the proliferation of cervical cancer cells was further validated in a colony formation assay, which showed significant reduction in the number of colonies formed by CRY1 siRNAtransfected Caski and SNU-17 cells (Figure 3C). Using a Boyden chamber coated with Matrigel, we determined the changes in cell invasion after $48 \mathrm{~h}$ of incubation. Compared with the control cells, the CRYI knock-down cells showed a significant decrease in invasion (Figure 3D).

Moreover, we analysed the functional role of $N A N O G$ in cervical cancer cells using siRNAs that targeted NANOG

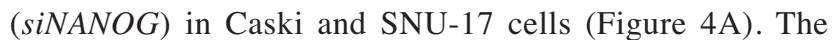
results revealed that silencing NANOG could significantly reduce cell proliferation (Figure 4B), colony formation (Figure 4C), and cell invasion (Figure 4D), as observed with CRY1 silencing. 
Table I. Association of CRY1 and NANOG expression with clinicopathological characteristics in cervical cancer.

\begin{tabular}{|c|c|c|c|c|c|c|}
\hline & No. & CRY1 & & No. & NANOG & \\
\hline & & Mean score $(95 \% \mathrm{CI})$ & $p$-Value & & Mean score $(95 \% \mathrm{CI})$ & $p$-Value \\
\hline All study subjects & 636 & 215.3 [210.7-219.9] & & 792 & $120.2[114.7-125.7]$ & \\
\hline \multicolumn{7}{|l|}{ Diagnostic category } \\
\hline Normal & 226 & $72.0[68.4-75.6]$ & $<0.001$ & 270 & 34.4 [31.9-36.9] & $<0.001$ \\
\hline Low-grade CIN & 73 & $118.0[110.3-125.6]$ & & 87 & $95.5[86.9-104.0]$ & \\
\hline High-grade CIN & 193 & $129.3[123.8-134.9]$ & & 265 & $169.5[163.5-175.5]$ & \\
\hline Cancer & 144 & $159.3[151.3-167.3]$ & & 170 & $192.4[184.6-200.3]$ & \\
\hline FIGO stage & & & 0.009 & & & 0.124 \\
\hline I & 97 & $151.9[142.1-161.8]$ & & 122 & 188.6 [179.4-197.9] & \\
\hline II-IV & 47 & $174.4[161.3-187.6]$ & & 48 & 202.1 [187.4-216.9] & \\
\hline Tumor grade & & & 0.821 & & & 0.285 \\
\hline Well/Moderate & 95 & $157.4[147.3-167.5]$ & & 97 & 186.8 [176.1-197.6] & \\
\hline Poor & 43 & $159.4[145.3-173.6]$ & & 56 & $196.4[182.4-210.4]$ & \\
\hline Cell type & & & 0.609 & & & 0.090 \\
\hline SCC & 119 & $158.3[150.0-166.6]$ & & 139 & 189.3 [180.4-198.2] & \\
\hline Others & 25 & $163.8[138.6-189.0]$ & & 31 & $206.7[191.0-222.3]$ & \\
\hline Tumor size & & & 0.131 & & & 0.524 \\
\hline$\leq 4 \mathrm{~cm}$ & 95 & $163.6[153.2-174.1]$ & & 122 & $194.0[185.0-203.0]$ & \\
\hline$>4 \mathrm{~cm}$ & 49 & 150.8 [138.8-162.7] & & 48 & $188.4[172.3-204.5]$ & \\
\hline LN metastasis & & & 0.447 & & & 0.428 \\
\hline No & 87 & $158.5[147.8-169.3]$ & & 113 & $190.3[180.7-199.8]$ & \\
\hline Yes & 26 & 149.9 [129.4-170.4] & & 25 & $199.4[177.0-221.7]$ & \\
\hline Chemoradiation response & & & 0.003 & & & 0.035 \\
\hline Good & 41 & 141.5 [126.1-156.9] & & 43 & $191.7[173.4-210.0]$ & \\
\hline $\mathrm{Bad}$ & 19 & $182.1[160.9-203.3]$ & & 21 & $221.3[207.8-234.9]$ & \\
\hline HPV test in CIN & & & 0.699 & & & 0.998 \\
\hline Negative & 20 & $121.6[105.5-137.6]$ & & 35 & 146.7 [128.4-164.9] & \\
\hline Positive & 188 & 125.1 [119.4-130.9] & & 244 & 146.6 [139.4-153.9] & \\
\hline
\end{tabular}

SCC: Squamous cell carcinoma; FIGO: International Federation of Gynecology and Obstetrics; LN metastasis: lymph node metastasis; HPV: human papillomavirus. Protein expression was determined by analysing an immunohistochemically-stained tissue array, as described in the Materials and Methods section.

Silencing of CRY1 enhanced cisplatin-induced apoptosis. Our results indicated that $C R Y 1$ and $N A N O G$ were interrelated in the poor response to chemoradiation treatment and prognosis; therefore, we analysed the relationship between CRY1 and NANOG expression using Spearman's rank correlation analysis in cervical cancer tissues and cell lines. Spearman's rank correlation results showed a significant positive correlation between CRY1 and NANOG expression in cervical neoplasia (Spearman's rho $=0.162, p=0.003$; Figure 5A). To assess the possible roles of $C R Y 1$ on the sensitivity of cervical cancer to cisplatin, Caski and SNU-17 cells were treated with various concentrations of cisplatin for 48 h. As shown in Figure 5B, cisplatin increased CRY1 protein expression in a dosedependent manner. Additionally, we observed up-regulation of cleaved-poly (ADP-ribose) polymerase (PARP) and cleavedcaspase 3 expression, which are valuable markers of apoptosis. To further investigate whether knock-down of CRY1 affects cisplatin sensitivity, we examined the apoptotic cell population by FACS-based Annexin-V/PI double staining (Figure 5C). The results revealed that siCRY1-transfected cells showed a significant increase in apoptosis compared to control cells in response to cisplatin, indicating that silencing of CRY1 enhanced cisplatin-induced apoptosis in cervical cancer cells. Intriguingly, knock-down of CRY1 elevated the percentage of apoptotic Caski cells from $15.87 \%$ to $27.18 \%$ and from $12.37 \%$ to $18.50 \%$ of apoptotic SNU-17 cells, relative to the control cells. To better understand the mechanism by which CRY1 regulates cell apoptosis, we examined the expression of several key regulators of apoptosis (Figure 5D). In siCRY1-transfected Caski and SNU-17 cells, several oncogenic proteins, including phosphorylated STAT3 at Tyr705 (pSTAT3) and NANOG, showed decreased expression. In contrast, the levels of p53 expression increased, suggesting that this pathway may be responsible for the CRY1-mediated anti-apoptotic effect.

\section{Discussion}

CRY1 is a member of the cryptochrome family, which is important for regulating the circadian feedback loop. Emerging evidence suggests that $C R Y 1$ disruption is related 
A

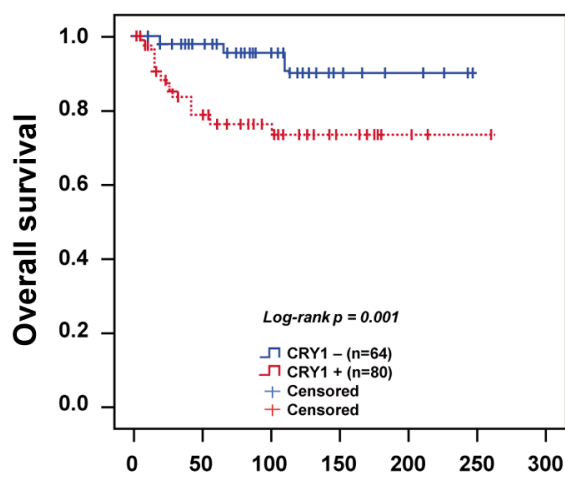

Months after initial treatment

D

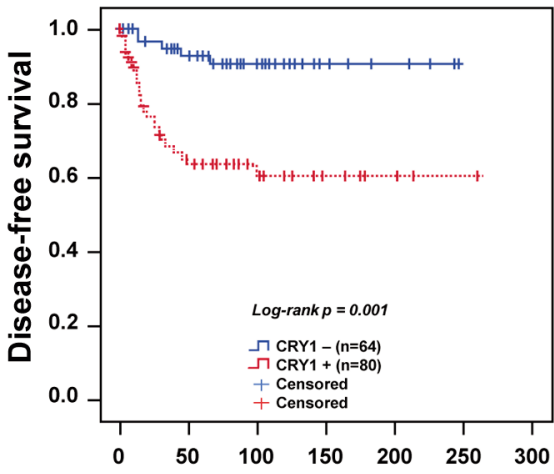

Months after initial treatment
B

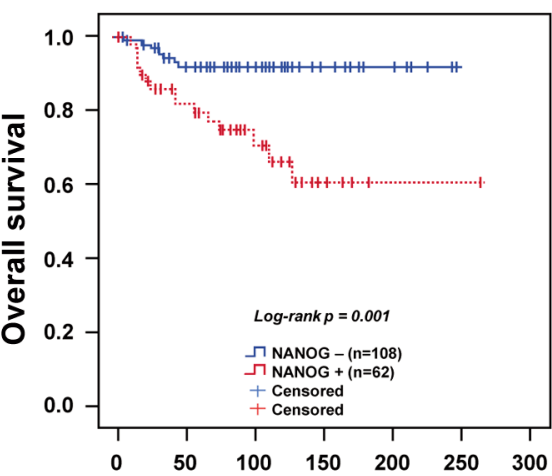

Months after initial treatment
C

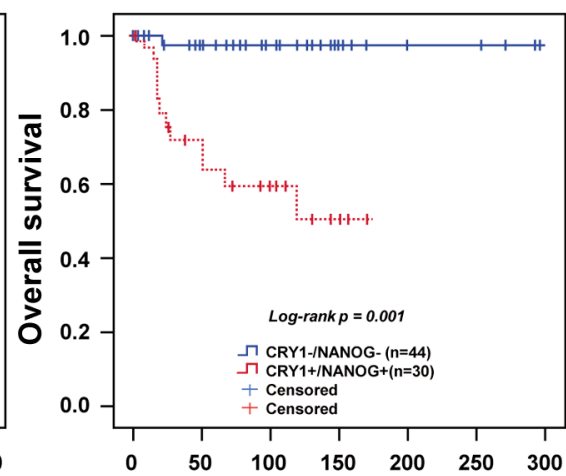

Months after initial treatment
E

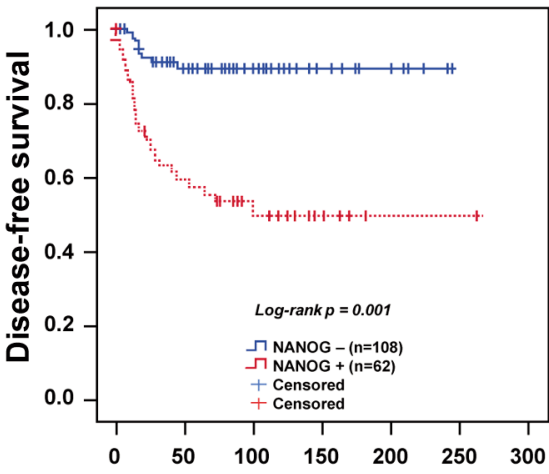

Months after initial treatment
$\mathbf{F}$

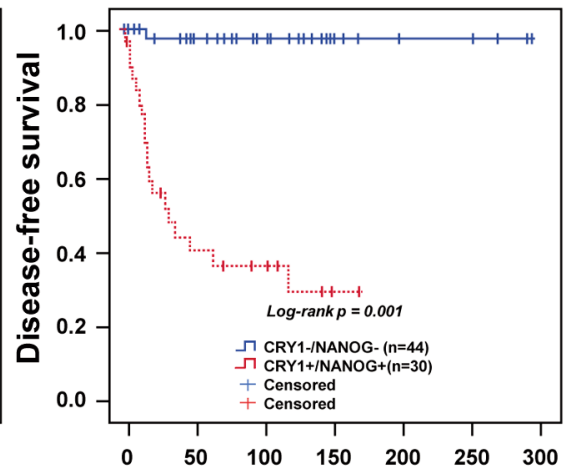

Months after initial treatment

Figure 2. Kaplan-Meier survival curves for CRY1 and NANOG expression in cervical cancer. Patients with cervical cancer over-expressing CRY1 showed worse $(A)$ overall survival $(p=0.001)$ and $(D)$ disease-free survival $(p=0.001)$ than those with low expression of CRY1. Cervical cancer patients with NANOG over-expression showed worse $(B)$ overall survival $(p=0.001)$ and $(E)$ disease-free survival $(p=0.001)$. Patients with overexpression of both CRY1 and NANOG showed worse $(C)$ overall survival $(p<0.001)$ and $(F)$ disease-free survival $(p<0.001)$ compared to patients with low expression of both CRY1 and NANOG.

Table II. Univariate and multivariate analyses of the associations between prognostic variables and overall survival and disease-free survival in cervical cancer.

\begin{tabular}{|c|c|c|c|c|}
\hline & \multicolumn{2}{|c|}{ Overall survival hazard ratio $[95 \% \mathrm{CI}], p$-Value } & \multicolumn{2}{|c|}{ Disease-free survival hazard ratio $[95 \% \mathrm{CI}], p$-Value } \\
\hline & Univariate & Multivariate & Univariate & Multivariate \\
\hline Age $(>50)$ & 0.78 [0.33-1.82], 0.567 & NA & $1.56[0.86-2.82], 0.141$ & NA \\
\hline FIGO stage (> IIA) & $4.47[1.98-10.07],<0.001$ & $2.59[0.70-9.53], 0.151$ & $6.12[3.19-11.75],<0.001$ & 3.38 [1.24-9.19], 0.017 \\
\hline Grade (poor) & $2.03[0.90-4.61], 0.090$ & NA & $1.64[0.90-2.99], 0.104$ & NA \\
\hline Cell type (non-SCC) & $2.47[1.06-5.78], 0.037$ & $4.93[1.18-20.56], 0.028$ & $1.06[0.49-2.28], 0.880$ & NA \\
\hline Tumor size $(>4 \mathrm{~cm})$ & $1.96[0.85-4.50], 0.114$ & NA & $2.18[1.19-.3 .99], 0.011$ & $0.86[0.30-2.43], 0.779$ \\
\hline LN metastasis & $2.68[1.05-6.84], 0.039$ & $1.81[0.56-5.83], 0.32$ & $4.23[2.11-8.45],<0.001$ & $2.78[1.01-7.63], 0.046$ \\
\hline $\mathrm{SCC} \mathrm{Ag}^{+}$ & $2.78[1.18-6.54], 0.020$ & $3.02[0.85-10.79], 0.87$ & $2.26[1.18-4.32], 0.014$ & $2.43[0.86-6.85], 0.091$ \\
\hline CRY $1^{+a}$ & $5.00[1.46-17.17], 0.011$ & $4.26[1.12-16.13], 0.033$ & $5.34[2.05-13.91], 0.001$ & $7.11[2.19-23.07], 0.001$ \\
\hline $\mathrm{NANOG}^{+\mathrm{b}}$ & 3.98 [1.64-9.66], 0.002 & $3.50[1.17-10.48], 0.024$ & $5.86[2.75-12.46],<0.001$ & $3.87[1.56-9.57], 0.003$ \\
\hline $\mathrm{CRY} 1^{+\mathrm{a}} / \mathrm{NANOG}^{+\mathrm{b}}$ & $20.66[2.67-159.16], 0.004$ & $11.17[1.36-91.16], 0.024$ & $40.01[5.35-300.34],<0.001$ & $18.68[2.34-149.00], 0.006$ \\
\hline
\end{tabular}

${ }^{a}$ Cut-off of CRY1 is over 221 of the immunohistochemistry (IHC) score; bCut-off value of NANOG+ is over 140 of the IHC score; CI: confidence interval; FIGO: International Federation of Gynecology and Obstetrics; LN: lymph node; NA: not applicable. 
A

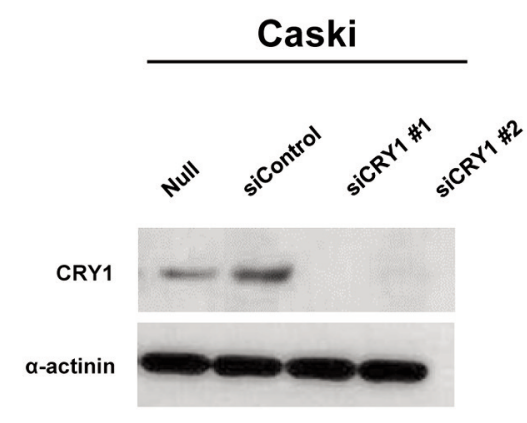

SNU-17

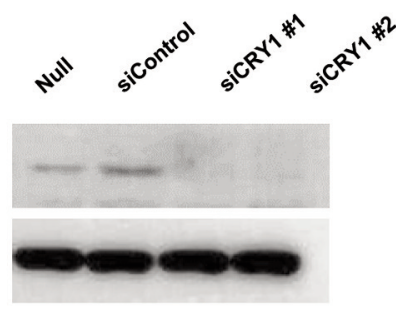

B

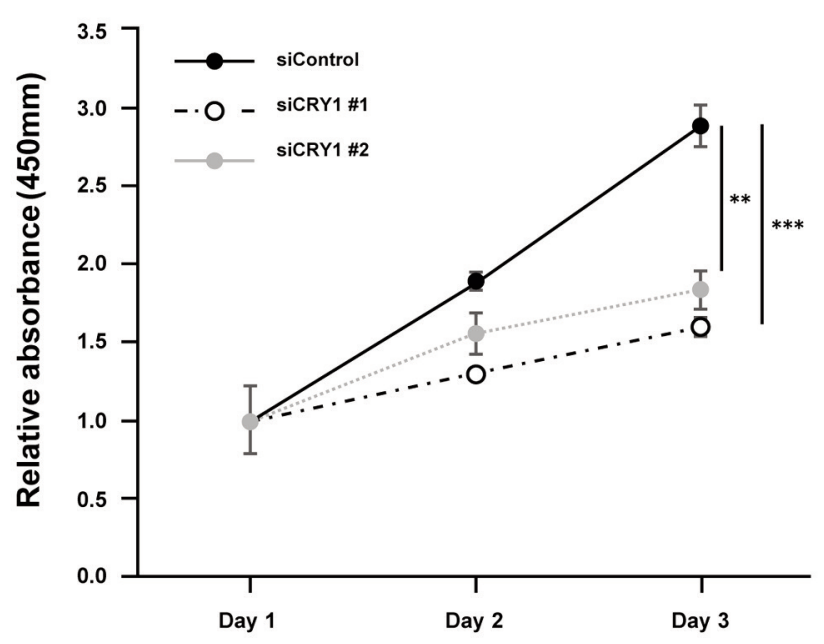

to carcinogenesis in various human cancers. However, to our knowledge, the role of CRY1 in cervical cancer is unexplored. Therefore, in this study, we evaluated the prognostic significance and functional role of CRY1 in cervical cancer using premalignant and malignant cervical cancer tissues and cell lines. Notably, we showed that poor response to chemotherapy was closely linked to apoptosis by suppressing STAT3 signalling, and activating p53 signalling with simultaneous upregulation of NANOG. To our knowledge, this is the first report on the association between $C R Y 1$ and NANOG in cervical cancer.

We evaluated the clinical and prognostic significance of $C R Y 1$ in cervical cancer, premalignant, and non-adjacent normal epithelial tissues. The results showed that CRY1 was over-expressed in cervical cancer tissues compared to premalignant and non-adjacent normal epithelial tissues. CRY1 over-expression was also significantly associated with advanced stage, poor chemoradiation responses, and poor OS and DFS in cervical cancer. A previous study has shown that CRY1 is over-expressed in gastric cancer tissues compared with normal tissues; this over-expression was associated with poor prognosis in patients with gastric cancer (24). CRY1 over-expression and its association with poor prognosis have also been observed in endometrial cancer, head and neck squamous cell carcinoma, and chronic myeloid leukaemia (25-27). Yu et al. have compared CRY1 expression between colorectal cancer tissues and adjacent non-cancerous tissues and found that over-expression of CRY1 in colorectal cancer tissues was correlated with more advanced stages, frequent LN metastasis, and poor patient outcomes (28). Additionally, they observed that CRY1 over-expression and its correlation with prognosis occurred more frequently in female patients. Similarly, CRY1 over-expression has been related to oestrogen receptor (ER) expression in breast cancer (29). In cervical cancer, the initiation site of carcinogenesis, the transformation zone, is very sensitive to ER, and ERmediated signalling disrupts several important pathways that are critical for carcinogenesis, such as apoptosis or DNA damage repair during the cell cycle in fact, ER reportedly oscillates in a circadian manner $(30,31)$. Therefore, in our 
C
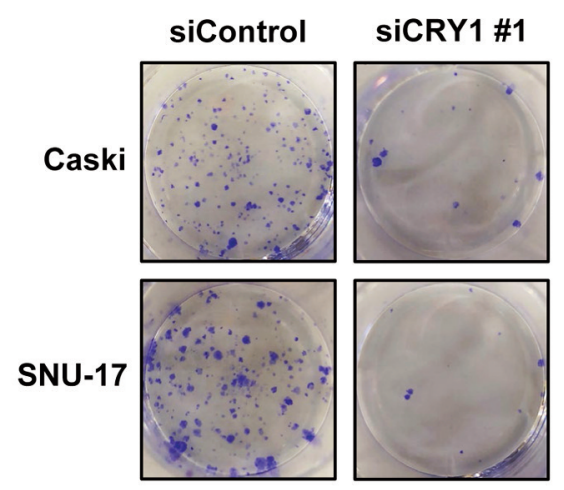

SiCRY1 \#2
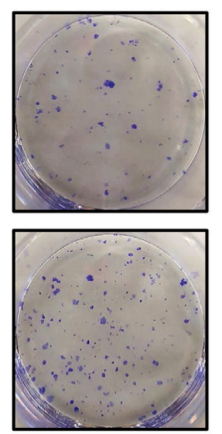

D
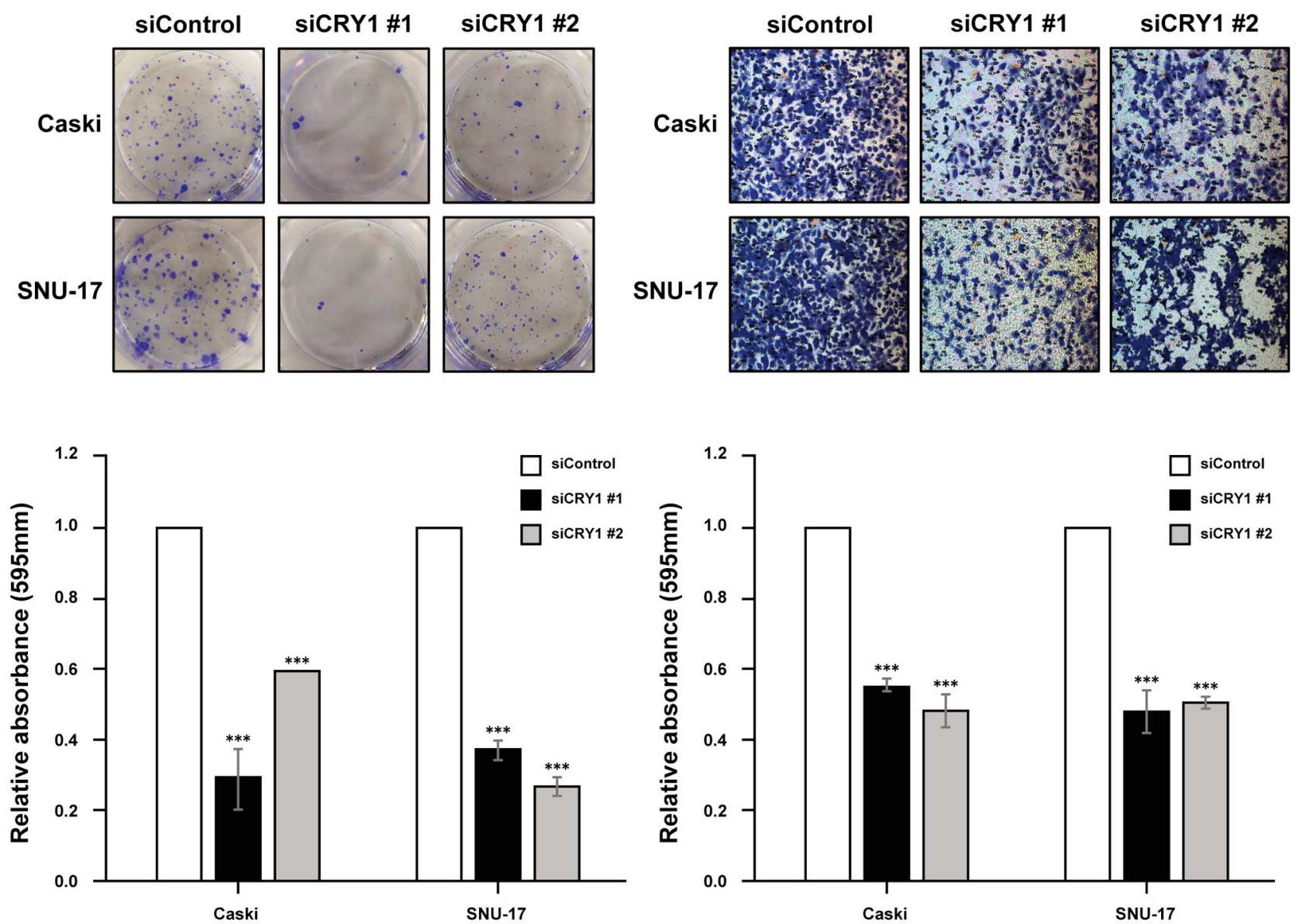

Figure 3. Effect of CRY1 in cervical cancer cell lines. CRY1 was knocked down in Caski and SNU-17 cells for 72 h. (A) Protein expression of CRY1 and $\alpha$-actinin was analysed by western blot. (B) The proliferation of siControl-and siCRY1-transfected Caski and SNU-17 cells was detected using EZ-Cytox assay, at indicated time points. (C) Colony formation was performed with siControl and siCRY1 transfected cell. Upper panel: representative image of colonogenic assay. Lower panel: quantitative results of colonogenic assay. (D) Cell invasion assay was conducted using the Boyden chamber assay. Upper panel: representative image of Boyden chamber assay. Lower panel: quantitative result of Boyden chamber assay. The number of asterisks $\left(^{*}\right)$ indicates the level of significance: $*^{*} p<0.05, * * * p<0.005$. Error bars represent the mean \pm standard error (S.E) of triplicate experiments.

future research, we plan to incorporate a subgroup analysis based on ER expression and explore the mechanisms by which ER modulates CRY1 expression in cervical cancer.

To explore the role of CRY1 in cervical cancer clinical specimens, we demonstrated that siRNA-mediated CRYI knock-down drastically inhibited cell viability in cervical cancer cell lines, Caski and SNU-17. Interestingly, in addition to its direct impact on tumour growth, we observed that CRY1 knock-down attenuated cisplatin resistance, as evidenced by a marked increase in the expression of prominent apoptotic markers, caspase-3, and PARP, in a dose-dependent manner in the cervical cancer cell lines. These data were further supported by a higher rate of apoptosis of $C R Y 1$ knock-down cells compared to control cells. Apoptosis refers to the process of programmed cell death, and dysregulation of the apoptotic mechanism is a sign of cancer development as well as cancer treatment resistance. Our study is in accordance with previous studies that have reported that a loss in the expression of CRY1 and $C R Y 2$ can sensitize tumour cells to apoptosis; however, to the best of our knowledge, the molecular link between apoptosis and circadian clock remains largely unknown (32, 33). With respect to the signalling pathways related to apoptosis, we compared the expression of STAT3, pSTAT3, and $p 53$ in control and $C R Y 1$ knock-down cervical cancer cell lines. Western blotting analysis revealed that pSTAT3 
A

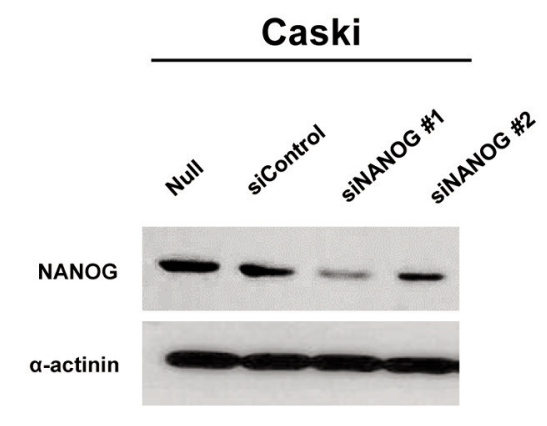

SNU-17

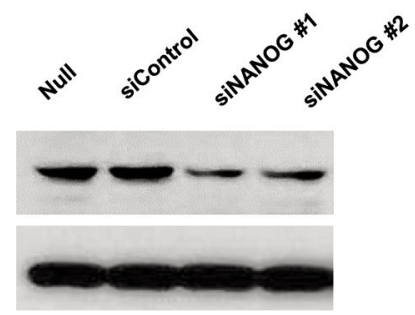

B

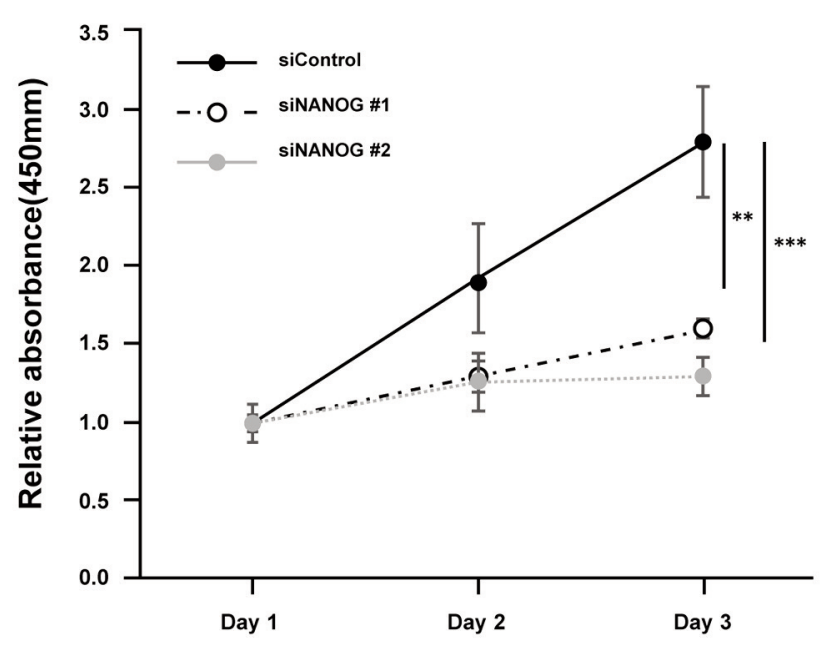

Caski

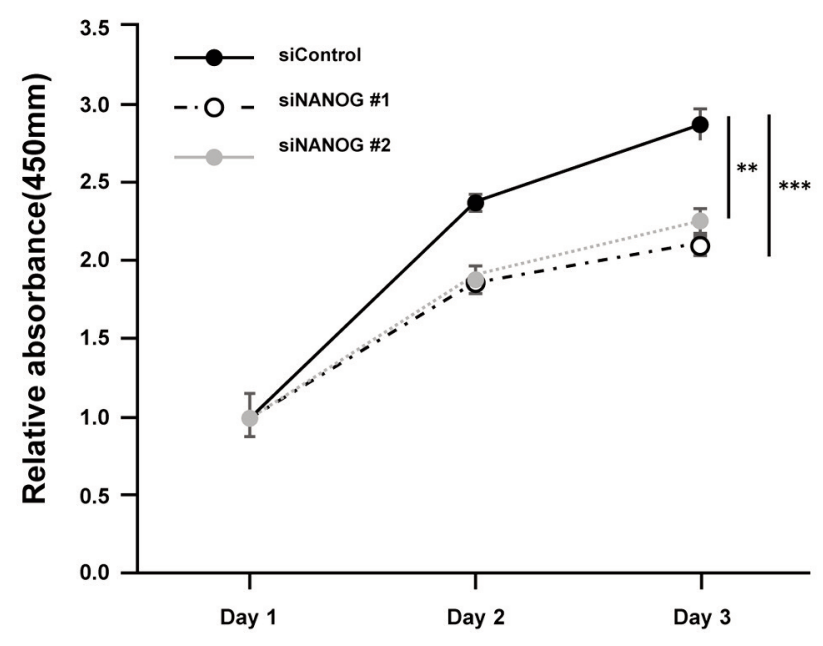

Figure 4. Continued expression was markedly reduced and p53 expression was increased in the CRY1 knock-down cervical cancer cells. A previous study has identified that in the clock-intrinsicapoptosis pathway, there appears to be a direct connection between the clock gene $C R Y 1$ and $p 53$ expression, indicating that $C R Y 1$ promotes $p 53$ degradation by facilitating the interaction of $p 53$ with MDM2 (34). Moreover, studies have shown that $p 53$ regulates STAT3 activity by inhibiting STAT3 phosphorylation, which subsequently inhibits STAT3 DNA binding activity; thus, functional loss of p53 results in the failure to dephosphorylate STAT3, thereby persistent STAT3 activation and chemotherapeutic resistance and tumour growth $(35,36)$. To the best of our knowledge, the association between CRY1, STAT3, and p53 has not been demonstrated previously in cervical cancer cell lines. Taken together, our findings suggest that as a clock oncogene, $C R Y 1$ could inhibit apoptosis via the p53/STAT3 signalling pathway in cervical cancer cells, which is an important signalling pathway for cell proliferation, invasion, metastasis, as well as chemoresistance in various cancers (37-39).
In addition to the role of CRYI in oncogenesis and chemoresistance in cancer, we further investigated its positive correlation with $N A N O G$, a key stem cell transcription factor, in cervical cancer specimens and cervical cancer cells. As previously reported, knockout of the circadian rhythm gene $B A M L 1$ reduces the development of murine skin tumours by reducing the number of tumour-initiating cells and enhancing the expression of tumour suppressor genes by modulating cancer stem cells (40). In breast cancer, Hadadi et al have demonstrated that chronic circadian disruption could promote increased expression of cancer stem cell associated genes and metastatic properties (41). Unfortunately, the molecular mechanism by which circadian rhythm genes regulate cancer stem cells is still not known. Per our knowledge, the correlation between the core circadian clock gene, $C R Y 1$, and NANOG has not been studied in cervical cancer. In our study, we investigated the positive correlation between CRYI and $N A N O G$. We found that $\operatorname{siCRY1}$ treatment resulted in the simultaneous down-regulation of NANOG. Moreover, CRY1 knock-down led to altered expression of other oncogenic 
C
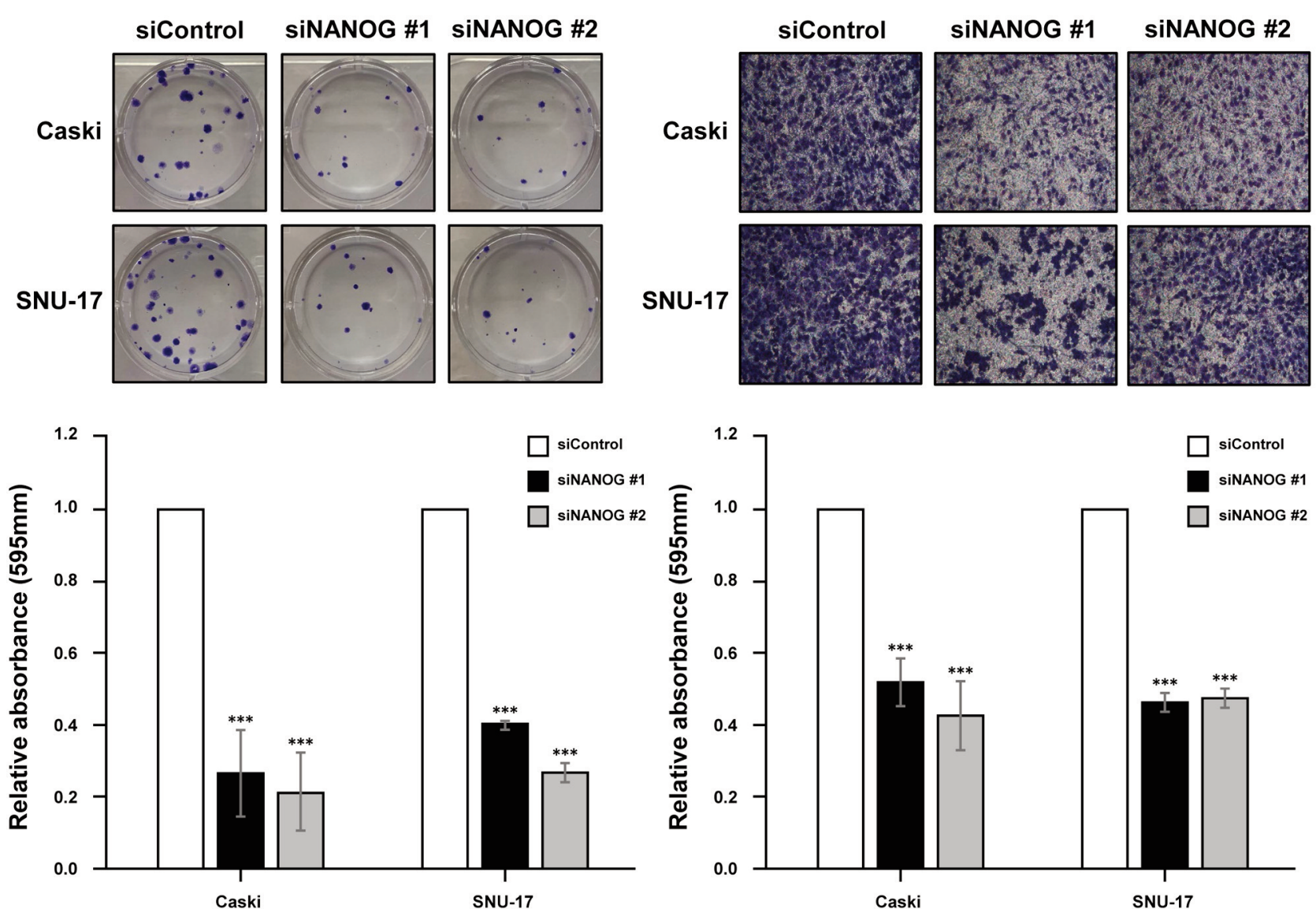

Figure 4. Effect of NANOG expression in cervical cancer cell lines. NANO. G was knocked down in Caski and SNU-17 cells for 72 (A) Protein expression of NANOG and $\alpha$-actinin was analysed by western blot. (B) The proliferation of siControl-and siNANOG-transfected Caski and SNU17 cells was detected using EZ-Cytox assay, at indicated time points. (C) Colony formation was performed with siControl and siCRY1 transfected cell. Upper panel: representative image of colonogenic assay. Lower panel: quantitative results of colonogenic assay. (D) Cell invasion assay was conducted using the Boyden chamber assay. Upper panel: representative image of Boyden chamber assay. Lower panel: quantitative result of Boyden chamber assay. The number of asterisks $\left(^{*}\right)$ indicates the level of significance: ${ }^{*} p<0.05, * * * p<0.005$. Error bars represent the mean \pm standard error (S.E) of triplicate experiments.

proteins, namely STAT3 and $p S T A T 3$. Importantly, pSTAT3, which is phosphorylated by $p 53$, binds to the $N A N O G$ promoter and influences its transcription in embryonic stem cells (ESCs) (42), liver cancer stem cells (43), and cervical cancer cells (44). Consequently, it is required for the transforming activity of STAT3, which in turn contributes to cell cycle progression and apoptosis (45). Therefore, based on previous reports and our results, we hypothesize that $C R Y 1$ may be hijacked by STAT3 via the $p 53$ signalling pathway to regulate $N A N O G$ expression, which participates in apoptosis and stemness in cervical cancer. However, further research is required to verify the role of the CRY1-STAT3-NANOG axis via p53 in cervical cancer cells.
In conclusion, we demonstrated that over-expression of CRY1 was significantly associated with advanced clinical stage, DFS, OS, as well as chemoresistance in patients with cervical cancer. Most importantly, this study revealed a positive correlation between the expression of CRY1 and NANOG, which could serve as a prognostic, predictive biomarker for patients with cervical cancer and aid in determining the response to chemoradiation therapy, which is crucial for suggesting candidate targets for developing a therapeutic strategy to overcome drug resistance. Though further studies are required to obtain convincing evidence to elucidate the underlying mechanisms, the data presented here suggest a novel role of CRYI in enhancing the chemoresistance in 
A

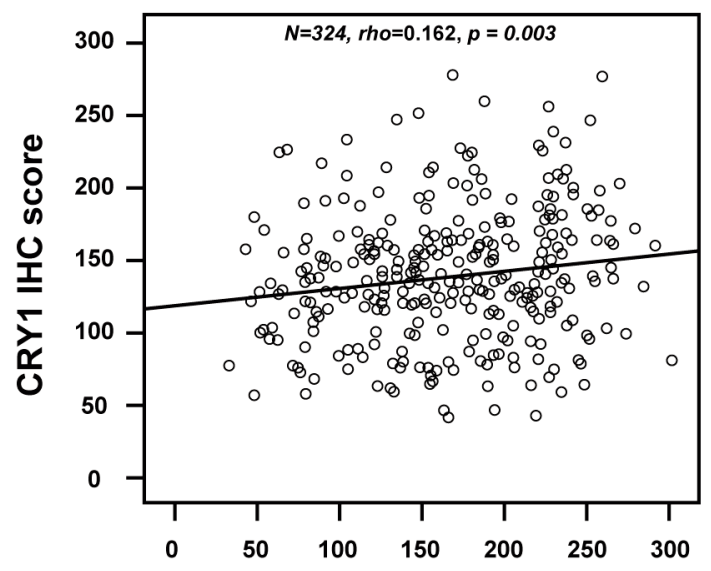

NANOG IHC scores

B

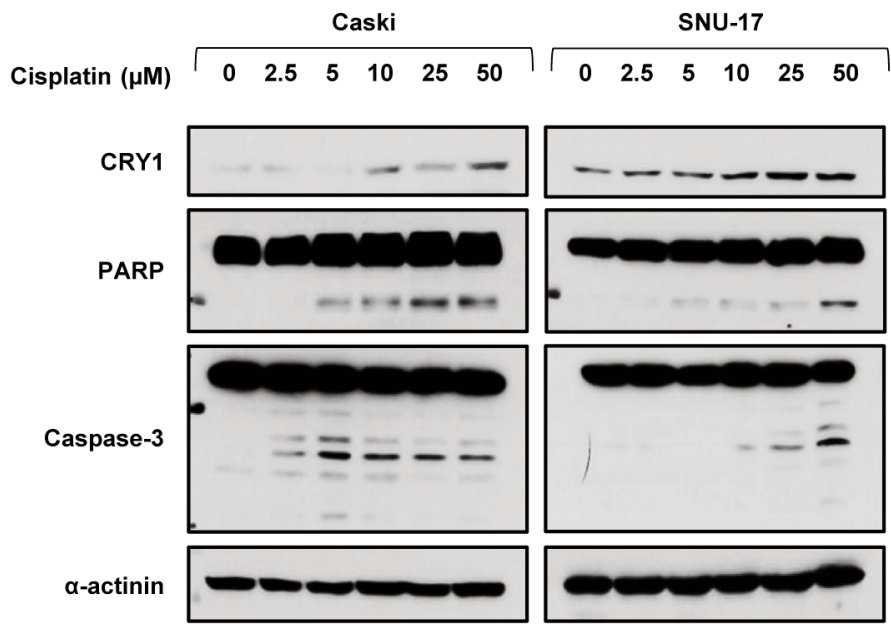

Figure 5. Continued

cervical cancer through the suppression of apoptosis. Finally, we suggest the involvement of the CRY1-STAT3-NANOG axis via 553 in cervical cancer.

\section{Conflicts of Interest}

The Authors declare no conflicts of interest in relation to this study.

\section{Authors' Contributions}

HC and J-YC designed and built tissue microarrays. GHH, HC, and $\mathrm{J}-\mathrm{YC}$ conceived and designed the study, and modified the experimental design. GHH and JK performed analysis of experimental or clinical data. HY performed functional studies. GHH and JK drafted the manuscript and figure legends. J-HK, J-YC, HC, and S.M.H revised the figures and added critical contents to the discussion, and were responsible for revising all portions of the submitted manuscript. All Authors read and approved the final manuscript.

\section{Acknowledgements}

This work was supported by the National Research Foundation of Korea (NRF) grant funded by the Korea government (MIST) (NRF2020R1A2C2004782). This research was supported by the Bio \& Medical Technology Development Program of the National Research Foundation (NRF) funded by the Korean government (MSIT) (NRF-2017M3A9B8 069610). This study was also supported by a faculty research grant of Yonsei University College of Medicine (No. 6-2020-0226).

\section{References}

1 Bray F, Ferlay J, Soerjomataram I, Siegel RL, Torre LA and Jemal A: Global cancer statistics 2018: GLOBOCAN estimates of incidence and mortality worldwide for 36 cancers in 185 countries. CA Cancer J Clin 68(6): 394-424, 2018. PMID: 30207593. DOI: $10.3322 /$ caac. 21492 


\section{C}

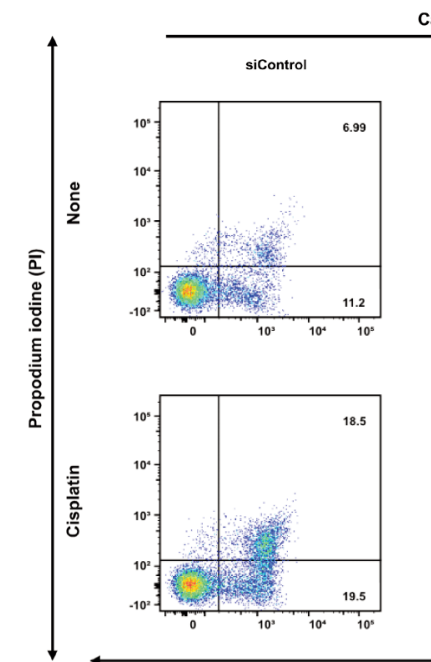

Caski
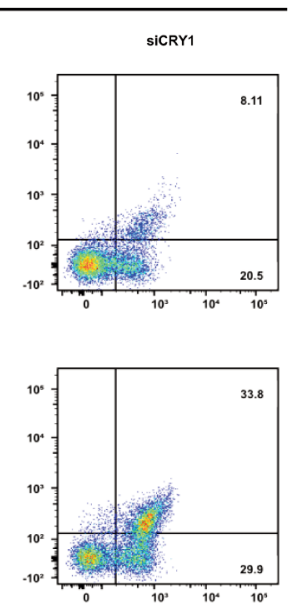

SNU-17
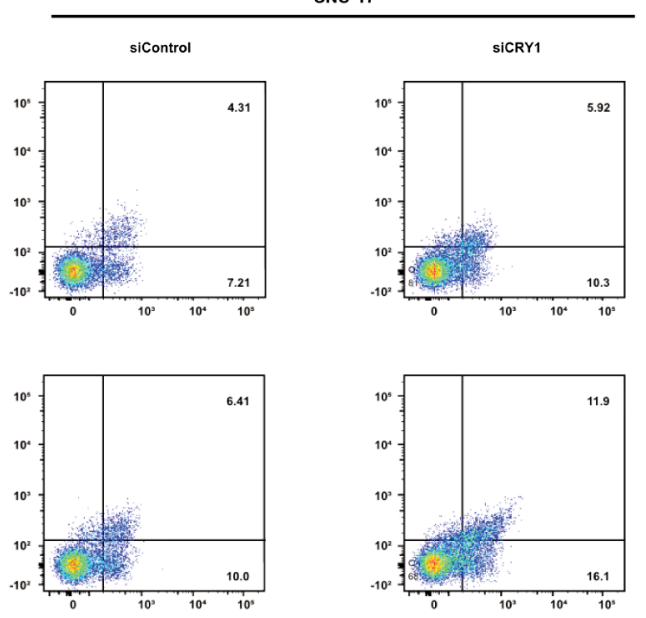

FITC Annexin V
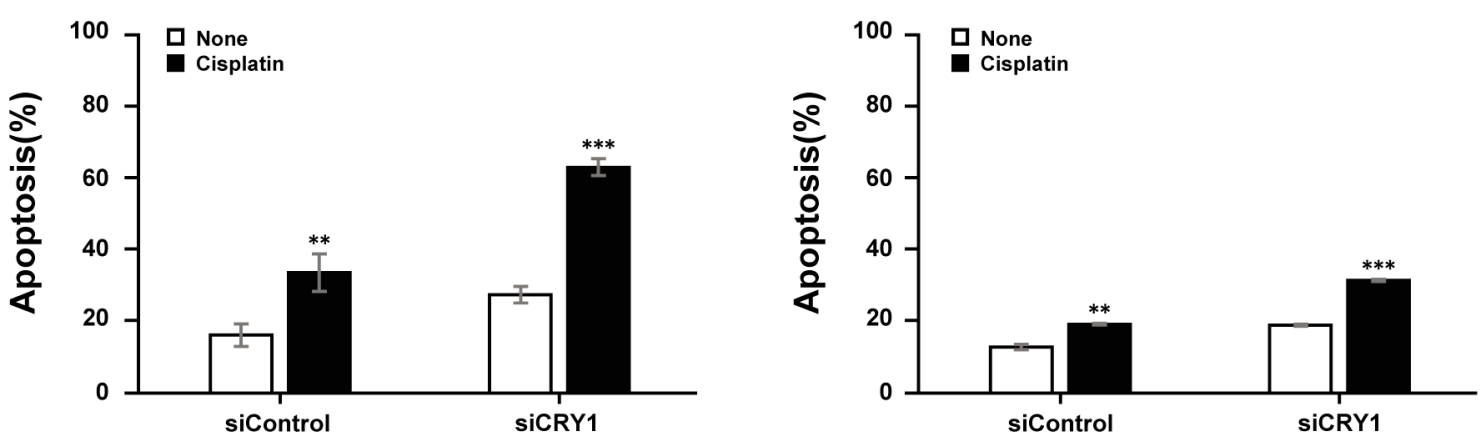

D

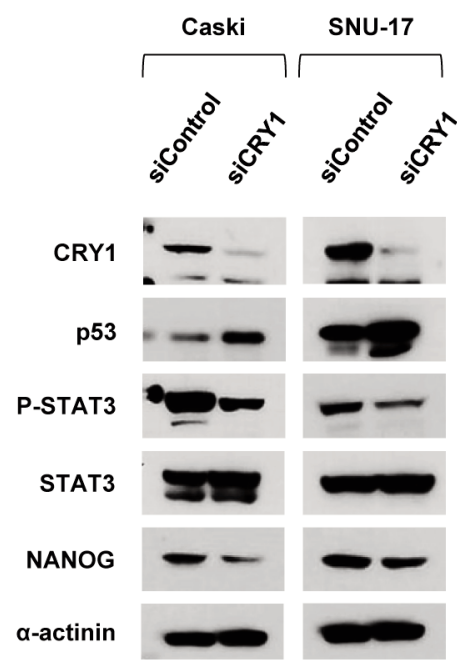

Figure 5. Effects of CRY1 in apoptosis and correlation with NANOG expression in cervical cancer specimens and cell lines. (A) Spearman's rank correlation analysis showed the positive correlation between CRY1 and NANOG expression in cervical cancer specimens (B) Caski and SNU-17 cells were treated with the indicated concentration of cisplatin for $48 \mathrm{~h}$. Cells were harvested; protein expression of CRY1, PARP, Caspase-3, and $\alpha$-actinin was analyzed by western blot. (C) CRY1 was knocked down in Caski and SNU-17 cells for 48 h and then cells were treated with $100 \mu M$ cisplatin for 48 h. Cells were then harvested and apoptosis was analyzed using flow cytometry after staining with annexin V-FITC/propidium iodide. Upper panel: representative scatter plots of propidium iodide (y-axis) versus annexin $V$ (x-axis). Lower panel: Quantitative analysis of the apoptotic cells. (D) CRY1 was knocked down in Caski and SNU-17 cells for $72 \mathrm{~h}$. Cells were then harvested and protein expression and activation status of CRY1, p53, P-STAT3, STAT3, NANOG, and $\alpha$-actinin were analysed by western blot. The number of asterisks $(*)$ indicates the level of significance: $* * p<0.05, * * * p<0.005$. Error bars represent mean \pm standard error (S.E) of triplicate experiments. 
2 Bray F, Carstensen B, Møller H, Zappa M, Zakelj MP, Lawrence $\mathrm{G}$, Hakama $\mathrm{M}$ and Weiderpass $\mathrm{E}$ : Incidence trends of adenocarcinoma of the cervix in 13 European countries. Cancer Epidemiol Biomarkers Prev 14(9): 2191-2199, 2005. PMID: 16172231. DOI: 10.1158/1055-9965.EPI-05-0231

3 Bray F, Loos AH, McCarron P, Weiderpass E, Arbyn M, Møller $\mathrm{H}$, Hakama $\mathrm{M}$ and Parkin DM: Trends in cervical squamous cell carcinoma incidence in 13 European countries: changing risk and the effects of screening. Cancer Epidemiol Biomarkers Prev 14(3): 677-686, 2005. PMID: 15767349. DOI: 10.1158/10559965.EPI-04-0569

4 Bartel DP: MicroRNAs: genomics, biogenesis, mechanism, and function. Cell 116(2): 281-297, 2004. PMID: 14744438. DOI: 10.1016/s0092-8674(04)00045-5

5 Eifel PJ, Winter K, Morris M, Levenback C, Grigsby PW, Cooper J, Rotman M, Gershenson D and Mutch DG: Pelvic irradiation with concurrent chemotherapy versus pelvic and paraaortic irradiation for high-risk cervical cancer: an update of radiation therapy oncology group trial (RTOG) 90-01. J Clin Oncol 22(5): 872-880, 2004. PMID: 14990643. DOI: 10.1200/ JCO.2004.07.197

6 Sulli G, Manoogian ENC, Taub PR and Panda S: Training the circadian clock, clocking the drugs, and drugging the clock to prevent, manage, and treat chronic diseases. Trends Pharmacol Sci 39(9): 812-827, 2018. PMID: 30060890. DOI: 10.1016/ j.tips.2018.07.003

7 Zhou M, Wang W, Karapetyan S, Mwimba M, Marqués J, Buchler NE and Dong X: Redox rhythm reinforces the circadian clock to gate immune response. Nature 523(7561): 472-476, 2015. PMID: 26098366. DOI: 10.1038/nature14449

8 Konopka RJ and Benzer S: Clock mutants of Drosophila melanogaster. Proc Natl Acad Sci USA 68(9): 2112-2116, 1971. PMID: 5002428. DOI: 10.1073/pnas.68.9.2112

9 Mattis J and Sehgal A: Circadian rhythms, sleep, and disorders of aging. Trends Endocrinol Metab 27(4): 192-203, 2016. PMID: 26947521. DOI: 10.1016/j.tem.2016.02.003

10 Khan S, Duan P, Yao L and Hou H: Shiftwork-mediated disruptions of circadian rhythms and sleep homeostasis cause serious health problems. Int J Genomics 2018: 8576890, 2018. PMID: 29607311. DOI: 10.1155/2018/8576890

11 Costa G, Haus E and Stevens R: Shift work and cancer considerations on rationale, mechanisms, and epidemiology. Scand J Work Environ Health 36(2): 163-179, 2010. PMID: 20126969. DOI: $10.5271 /$ sjweh.2899

12 Davis S, Mirick DK and Stevens RG: Night shift work, light at night, and risk of breast cancer. J Natl Cancer Inst 93(20): 1557-1562, 2001. PMID: 11604479. DOI: 10.1093/jnci/ 93.20 .1557

13 Lee JH and Sancar A: Regulation of apoptosis by the circadian clock through NF-kappaB signaling. Proc Natl Acad Sci USA 108(29): 12036-12041, 2011. PMID: 21690409. DOI: 10.1073/ pnas. 1108125108

14 Hardin PE: Molecular genetic analysis of circadian timekeeping in Drosophila. Adv Genet 74: 141-173, 2011. PMID: 21924977. DOI: 10.1016/B978-0-12-387690-4.00005-2

15 Habashy DM, Eissa DS and Aboelez MM: Cryptochrome-1 gene expression is a reliable prognostic indicator in egyptian patients with chronic lymphocytic leukemia: a prospective cohort study. Turk J Haematol 35(3): 168-174, 2018. PMID: 28884705. DOI: 10.4274/tjh.2017.0169
16 Mazzoccoli G, Piepoli A, Carella M, Panza A, Pazienza V, Benegiamo G, Palumbo O and Ranieri E: Altered expression of the clock gene machinery in kidney cancer patients. Biomed Pharmacother 66(3): 175-179, 2012. PMID: 22436651. DOI: 10.1016/j.biopha.2011.11.007

17 Pazienza V, Piepoli A, Panza A, Valvano MR, Benegiamo G, Vinciguerra M, Andriulli A and Mazzoccoli G: SIRT1 and the clock gene machinery in colorectal cancer. Cancer Invest 30(2): 98-105, 2012. PMID: 22149272. DOI: 10.3109/07357907.2011.640650

18 Zhou L, Yu Y, Sun S, Zhang T and Wang M: Cry 1 regulates the clock gene network and promotes proliferation and migration via the Akt/P53/P21 pathway in human osteosarcoma cells. J Cancer 9(14): 2480-2491, 2018. PMID: 30026846. DOI: 10.7150/jca.25213

19 Zieker D, Jenne I, Koenigsrainer I, Zdichavsky M, Nieselt K, Buck K, Zieker J, Beckert S, Glatzle J, Spanagel R, Koenigsrainer A, Northoff $\mathrm{H}$ and Loeffler $\mathrm{M}$ : Circadian expression of clock- and tumor suppressor genes in human oral mucosa. Cell Physiol Biochem 26(2): 155-166, 2010. PMID: 20798499. DOI: $10.1159 / 000320547$

20 Koyanagi S, Kuramoto Y, Nakagawa H, Aramaki H, Ohdo S, Soeda $\mathrm{S}$ and Shimeno H: A molecular mechanism regulating circadian expression of vascular endothelial growth factor in tumor cells. Cancer Res 63(21): 7277-7283, 2003. PMID: 14612524.

21 Therasse P, Arbuck SG, Eisenhauer EA, Wanders J, Kaplan RS, Rubinstein L, Verweij J, Van Glabbeke M, van Oosterom AT, Christian MC and Gwyther SG: New guidelines to evaluate the response to treatment in solid tumors. European Organization for Research and Treatment of Cancer, National Cancer Institute of the United States, National Cancer Institute of Canada. J Natl Cancer Inst 92(3): 205-216, 2000. PMID: 10655437. DOI: 10.1093/jnci/92.3.205

22 Puram RV, Kowalczyk MS, de Boer CG, Schneider RK, Miller PG, McConkey M, Tothova Z, Tejero $\mathrm{H}$, Heckl D, Järås $M$, Chen MC, Li H, Tamayo A, Cowley GS, Rozenblatt-Rosen O, Al-Shahrour F, Regev A and Ebert BL: Core circadian clock genes regulate leukemia stem cells in AML. Cell 165(2): 303316, 2016. PMID: 27058663. DOI: 10.1016/j.cell.2016.03.015

23 Dong Z, Zhang G, Qu M, Gimple RC, Wu Q, Qiu Z, Prager BC, Wang X, Kim LJY, Morton AR, Dixit D, Zhou W, Huang H, Li B, Zhu Z, Bao S, Mack SC, Chavez L, Kay SA and Rich JN: Targeting glioblastoma stem cells through disruption of the circadian clock. Cancer Discov 9(11): 1556-1573, 2019. PMID: 31455674. DOI: 10.1158/2159-8290.CD-19-0215

$24 \mathrm{Hu}$ ML, Yeh KT, Lin PM, Hsu CM, Hsiao HH, Liu YC, Lin HY, Lin SF and Yang MY: Deregulated expression of circadian clock genes in gastric cancer. BMC Gastroenterol 14: 67, 2014. PMID: 24708606. DOI: $10.1186 / 1471-230 X-14-67$

25 Hsu CM, Lin SF, Lu CT, Lin PM and Yang MY: Altered expression of circadian clock genes in head and neck squamous cell carcinoma. Tumour Biol 33(1): 149-155, 2012. PMID: 22081375. DOI: $10.1007 / \mathrm{s} 13277-011-0258-2$

26 Yang MY, Chang JG, Lin PM, Tang KP, Chen YH, Lin HY, Liu TC, Hsiao HH, Liu YC and Lin SF: Downregulation of circadian clock genes in chronic myeloid leukemia: alternative methylation pattern of hPER3. Cancer Sci 97(12): 1298-1307, 2006. PMID: 16999817. DOI: 10.1111/j.1349-7006.2006.00331.x

27 Yang MY, Yang WC, Lin PM, Hsu JF, Hsiao HH, Liu YC, Tsai HJ, Chang CS and Lin SF: Altered expression of circadian clock genes in human chronic myeloid leukemia. J Biol Rhythms 
26(2): 136-148, 2011. PMID: 21454294. DOI: 10.1177/0748 730410395527

28 Yu H, Meng X, Wu J, Pan C, Ying X, Zhou Y, Liu R and Huang W: Cryptochrome 1 overexpression correlates with tumor progression and poor prognosis in patients with colorectal cancer. PLoS One 8(4): e61679, 2013. PMID: 23626715. DOI: 10.1371/journal.pone.0061679

29 Lesicka M, Jabłońska E, Wieczorek E, Seroczyńska B, Siekierzycka A, Skokowski J, Kalinowski L, Wąsowicz W and Reszka E: Altered circadian genes expression in breast cancer tissue according to the clinical characteristics. PLoS One 13(6): e0199622, 2018. PMID: 29958276. DOI: 10.1371/journal. pone.0199622

30 den Boon JA, Pyeon D, Wang SS, Horswill M, Schiffman M, Sherman M, Zuna RE, Wang Z, Hewitt SM, Pearson R, Schott M, Chung L, He Q, Lambert P, Walker J, Newton MA, Wentzensen N and Ahlquist P: Molecular transitions from papillomavirus infection to cervical precancer and cancer: Role of stromal estrogen receptor signaling. Proc Natl Acad Sci USA 112(25): E3255E3264, 2015. PMID: 26056290. DOI: 10.1073/pnas.1509322112

31 Rossetti S, Esposito J, Corlazzoli F, Gregorski A and Sacchi N: Entrainment of breast (cancer) epithelial cells detects distinct circadian oscillation patterns for clock and hormone receptor genes. Cell Cycle 11(2): 350-360, 2012. PMID: 22193044. DOI: 10.4161/cc.11.2.18792

32 Fuchs $\mathrm{Y}$ and Steller H: Programmed cell death in animal development and disease. Cell 147(4): 742-758, 2011. PMID: 22078876. DOI: 10.1016/j.cell.2011.10.033

33 Wong RS: Apoptosis in cancer: from pathogenesis to treatment. J Exp Clin Cancer Res 30: 87, 2011. PMID: 21943236. DOI: 10.1186/1756-9966-30-87

34 Jia M, Su B, Mo L, Qiu W, Ying J, Lin P, Yang B, Li D, Wang $\mathrm{D}, \mathrm{Xu} \mathrm{L}, \mathrm{Li} \mathrm{H}$, Zhou Z, Li X and Li J: Circadian clock protein CRY1 prevents paclitaxel induced senescence of bladder cancer cells by promoting p53 degradation. Oncol Rep 45(3): 1033 1043, 2021. PMID: 33650658. DOI: 10.3892/or.2020.7914

35 Wörmann SM, Song L, Ai J, Diakopoulos KN, Kurkowski MU, Görgülü K, Ruess D, Campbell A, Doglioni C, Jodrell D, Neesse A, Demir IE, Karpathaki AP, Barenboim M, Hagemann T, RoseJohn S, Sansom O, Schmid RM, Protti MP, Lesina M and Algül $\mathrm{H}$ : Loss of P53 function activates JAK2-STAT3 signaling to promote pancreatic tumor growth, stroma modification, and gemcitabine resistance in mice and is associated with patient survival. Gastroenterology 151(1): 180-193.e12, 2016. PMID: 27003603. DOI: $10.1053 /$ j.gastro.2016.03.010

36 Ozturk N, Lee JH, Gaddameedhi S and Sancar A: Loss of cryptochrome reduces cancer risk in p53 mutant mice. Proc Natl Acad Sci USA 106(8): 2841-2846, 2009. PMID: 19188586. DOI: $10.1073 /$ pnas .0813028106
37 Avalle L, Camporeale A, Camperi A and Poli V: STAT3 in cancer: A double edged sword. Cytokine 98: 42-50, 2017. PMID: 28579221. DOI: 10.1016/j.cyto.2017.03.018

38 Banerjee $\mathrm{K}$ and Resat $\mathrm{H}$ : Constitutive activation of STAT3 in breast cancer cells: A review. Int J Cancer 138(11): 2570-2578, 2016. PMID: 26559373. DOI: 10.1002/ijc. 29923

$39 \mathrm{Yu} \mathrm{H}$ and Jove R: The STATs of cancer--new molecular targets come of age. Nat Rev Cancer 4(2): 97-105, 2004. PMID: 14964307. DOI: $10.1038 / \mathrm{nrc} 1275$

40 Janich P, Pascual G, Merlos-Suárez A, Batlle E, Ripperger J, Albrecht U, Cheng HY, Obrietan K, Di Croce L and Benitah SA: The circadian molecular clock creates epidermal stem cell heterogeneity. Nature 480(7376): 209-214, 2011. PMID: 22080954. DOI: $10.1038 /$ nature 10649

41 Hadadi E, Taylor W, Li XM, Aslan Y, Villote M, Rivière J, Duvallet G, Auriau C, Dulong S, Raymond-Letron I, Provot S, Bennaceur-Griscelli A and Acloque H: Chronic circadian disruption modulates breast cancer stemness and immune microenvironment to drive metastasis in mice. Nat Commun 11(1): 3193, 2020. PMID: 32581213. DOI: 10.1038/s41467-02016890-6

42 Lee TK, Castilho A, Cheung VC, Tang KH, Ma S and Ng IO: CD24(+) liver tumor-initiating cells drive self-renewal and tumor initiation through STAT3-mediated NANOG regulation. Cell Stem Cell 9(1): 50-63, 2011. PMID: 21726833. DOI: 10.1016/j.stem.2011.06.005

43 Hosseinpour B, Bakhtiarizadeh MR, Khosravi P and Ebrahimie E: Predicting distinct organization of transcription factor binding sites on the promoter regions: a new genome-based approach to expand human embryonic stem cell regulatory network. Gene 531(2): 212-219, 2013. PMID: 24042128. DOI: 10.1016/ j.gene.2013.09.011

44 Wang H, Cai HB, Chen LL, Zhao WJ, Li P, Wang ZQ and Li Z: STAT3 correlates with stem cell-related transcription factors in cervical cancer. J Huazhong Univ Sci Technolog Med Sci 35(6): 891-897, 2015. PMID: 26670442. DOI: 10.1007/s11596-0151524-0

45 Bowman T, Broome MA, Sinibaldi D, Wharton W, Pledger WJ, Sedivy JM, Irby R, Yeatman T, Courtneidge SA and Jove R: Stat3mediated Myc expression is required for Src transformation and PDGF-induced mitogenesis. Proc Natl Acad Sci USA 98(13): 73197324, 2001. PMID: 11404481. DOI: 10.1073/pnas.131568898

Received July 20, 2021

Revised August 12, 2021 Accepted August 23, 2021 